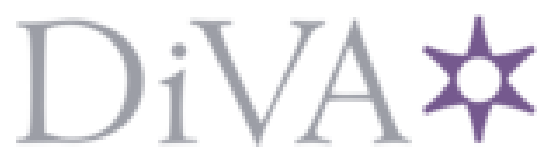

http://www.diva-portal.org

\title{
Postprint
}

This is the accepted version of a paper published in Journal of Neurotrauma. This paper has been peer-reviewed but does not include the final publisher proof-corrections or journal pagination.

Citation for the original published paper (version of record):

Darragh, W., Zhou, Z., Li, X., Jamie, K., David, N. et al. (2021)

Mechanical Properties of the Cranial Meninges: A Systematic Review

Journal of Neurotrauma, 38(13): 1748-1761

Access to the published version may require subscription.

N.B. When citing this work, cite the original published paper.

Permanent link to this version:

http://urn.kb.se/resolve?urn=urn:nbn:se:kth:diva-297597 


\section{Mechanical Properties of the Cranial Meninges: A Systematic Review}

Article in Journal of Neurotrauma · November 2020

DOI: $10.1089 /$ neu. 2020.7288

CITATIONS

6 authors, including:

Darragh Walsh

University of Limerick

5 PUBLICATIONS 11 CITATIONS

SEE PROFILE

Xiaogai Li

KTH Royal Institute of Technology

71 PUBLICATIONS 457 CITATIONS

SEE PROFILE

Some of the authors of this publication are also working on these related projects:

Project $\quad$ Fitness to drive in cognitive impairment: A quantitative study of GPs experience View project

Project Clinical Applications of Finite Element \& Neuroimaging Techniques for Improved Patient Treatment View project
READS

192






\section{Title}

Mechanical properties of the Cranial Meninges; A Systematic Review

\section{Author Information}

Darragh R. Walsh",a,b, Zhou Zhou ${ }^{c}$, Xiaogai Lic, Jamie Kearns ${ }^{d}$, David T. Newporta,b, John J.E. Mulvihill $^{2 a, b, e}$

${ }^{1}$ First author

${ }^{2}$ Corresponding author

a Bernal Institute, University of Limerick, Limerick, Ireland

b School of Engineering, University of Limerick, Limerick, Ireland

${ }^{c}$ Division of Neuronic Engineering, KTH Royal Institute of Technology, Huddinge, Sweden

${ }^{\mathrm{d}}$ Munster Rugby High Performance Centre, University of Limerick, Limerick, Ireland

e Health Research Institute, University of Limerick, Limerick, Ireland 


\section{Abstract}

The meninges are membranous tissues which are pivotal in maintaining homeostasis of the central nervous system. Despite the importance of the cranial meninges in nervous system physiology and in head injury mechanics, our knowledge of the tissues' mechanical behaviour and structural composition is limited. This systematic review analyses the existing literature on the mechanical properties of the meningeal tissues. Publications were identified from a search of Scopus, Academic Search Complete and Web of Science and screened for eligibility according to PRISMA guidelines. The review details the wide range of testing techniques employed to date and the significant variability in the observed experimental findings. Our findings identify many gaps in the current literature which can serve as a guide for future work for meningeal mechanics investigators. The review identifies no peer-reviewed mechanical data on the falx and tentorium tissues, both of which have been identified as key structures in influencing brain injury mechanics. A dearth of mechanical data for the pia-arachnoid complex was also identified (no experimental mechanics studies on the human pia-arachnoid complex were identified), which is desirable for biofidelic modelling of human head injuries. Finally, this review provides recommendations on how experiments can be conducted to allow for standardisation of test methodologies, enabling simplified comparisons and conclusions on meningeal mechanics.

\section{Keywords}

Dura mater; Pia-arachnoid complex (PAC); Falx cerebri; Dural graft design; TBI Finite element modelling; Injury mechanics 


\section{Introduction}

The meninges are membranous tissues that encase the central nervous system. ${ }^{1}$ The cranial meninges maintain homeostasis of the central nervous system by providing mechanical, immunological and vascular support to the brain parenchyma.$^{2,3}$ Studies have begun to elucidate the key role of the meninges in modulating the immune response to pathologies within the central nervous system (CNS), with research demonstrating the capacity of the meningeal tissue to migrate immune cells from the calvarium to the brain following stroke ${ }^{4}$ and their ability to support robust inflammatory reactions following traumatic injury. ${ }^{5}$ However, the tissues have conventionally been overlooked as passive, inert sacs $^{2,3}$ and thus, our knowledge of fundamental meningeal anatomy and physiology is limited. ${ }^{6}$

The meninges frequently require reconstruction utilising dural graft biomaterials. Dura-mimetic biomaterials are required to repair dural defects resulting from a number of causes including surgical procedures of the cranium which remove sections of dura to access the parenchymal tissue, penetrative traumatic injuries and congenital abnormalities. ${ }^{7}$ These biomaterials should match native tissue compliance and stiffness to prevent postoperative complications ${ }^{8,9}$ and thus, knowledge of native meningeal tissue mechanical properties are desired for novel graft biomaterial design.

Damage to the meninges is also a frequent clinical observation associated with traumatic brain injury (TBI). 5, 10 While estimates vary drastically, it is predicted that approximately 50 million individuals sustain a TBI each year, and that about half the world's population will suffer at least one TBI in their lifetime. ${ }^{11}$ Our currently limited understanding of the mechanisms of $\mathrm{TBI}$ is a major limitation in the development of more effective TBI prevention and treatment strategies. ${ }^{12}$

Conventionally, TBI research has primarily focused on the mechanics and pathophysiological cascade of brain tissue. Computational modelling of head impacts represents a promising tool to uncover the still debated mechanistic role of the various intracranial tissues in the etiology of 
TBI. ${ }^{13}$ Finite element (FE) analyses of injury-level impacts have provided invaluable insights into the mechanics of TBI and has highlighted the propensity for rotational acceleration of the head, in particular, to induce deleterious strains within the brain. ${ }^{14-16}$ However, both experimental ${ }^{17,} 18$ and computational ${ }^{19-21}$ studies suggest that both the dura mater and the leptomeninges protect the brain against TBI. A combined approach of experimental indentation and FE analysis identified that the meninges reduce the magnitudes of strain in the brain by up to $65 \%$ in indentation-type deformations. ${ }^{17}$ A computational modelling study by Gu et al (2012) focussed on the influence of the meninges in damping the dynamic response of the brain to primary blast waves. ${ }^{22}$ The authors identified that when the composite meninges were excluded from their model, the peak principal strain observed in the brain increased by 2.5 -fold. Modelling work by Scott et al (2015), which simulated porcine head rotation injuries, found that inter-subject variations in porcine meningeal microstructure resulted in a reduction of injurious skull-brain displacement by up to $28 \% .{ }^{20}$ Conversely, the meninges also appear to mediate the propagation of deleterious impact-induced TBI loads to the cortex via the falx cerebri. ${ }^{13,23} \mathrm{~A}$ computational model exploring the role of the falx in TBI illustrated that addition of the falx to a head model resulted in a 2-fold increase in strain within the corpus callosum of the brain. ${ }^{13}$ Thus, it appears that a holistic approach, wherein the contribution of all tissues of the head are considered, is required in the study of TBI.

This review conducts a systematic review of the literature investigating the mechanical properties of various regions of the cranial meninges. First, a description of meningeal anatomy, physiology and structural architecture is provided for context, followed by a section that explains in lay terms the mechanical properties that are discussed in this review. We then provide the first review of meningeal mechanics investigations to date. This analysis will aid researchers in making the appropriate choice of meningeal material properties for biofidelic clinical and computational modelling efforts. 


\section{Anatomy and Physiology of the Meninges}

Knowledge of the anatomy and physiology of the meninges is required to understand the tissues' mechanical behaviour. This section provides a brief overview of the anatomy and physiology of the meningeal regions discussed in this review. An illustration of the anatomy of the meninges is provided for context in Fig. 1.

\section{Dura Mater}

The dura mater (pachymeninx) is the outermost layer of the meninges and adheres directly to the periosteum of the skull in many regions ${ }^{24}$ Structurally, the dura is a connective tissue with a dense collagen architecture..$^{25-27}$ The dura mater has a concentrated network of vasculature, which supports cranial immune cell traffic. ${ }^{2}$ The dura is also a highly innervated tissue and is thought to contain the majority of the recently discovered meningeal lymphatic vessels. ${ }^{28}$

\section{Falx Cerebri and Tentorium Cerebelli}

The falx cerebri, the largest of the dural extensions, is present in the longitudinal cerebral fissure and is responsible for partitioning the left and right cerebral hemispheres ${ }^{29}$ (see Fig. 1). It has been proposed that the function of the falx is to constrain brain displacement and rotation within the cranial cavity. ${ }^{30},{ }^{31}$ The tentorium cerebelli, the second largest extension, separates the cerebellum and cerebral hemispheres. ${ }^{29}$ The most probable function of the tentorium is to support the weight of the cerebral hemispheres..$^{31,32}$

\section{Pia-Arachnoid Complex (PAC)}

The PAC, also known as the leptomeninges, is an intricate structure in which the pia and arachnoid layers are connected with trabeculae composed of collagen bundles known as arachnoid trabeculae. The PAC is a relatively thin structure when compared to the thick dural 
membrane. Between the pia and arachnoid layers, there is a 'subarachnoid space' which contains cerebrospinal fluid and subarachnoid vasculature. ${ }^{21}$ Similar to the dura mater, the pia and arachnoid play important roles in cerebrovascular circulation. ${ }^{33}$ Along with the structure's immunological functionality, the PAC is thought to assist in dissipating harmful energy associated with rapid head movement. ${ }^{21,34}$

\section{Structural architecture of the meninges}

Knowledge of a tissue's architecture and structural alignment is required to understand its mechanical behaviour. This section discusses the structural characteristics of the cranial meninges.

\section{Dura mater structural composition and alignment}

The collagenic architecture of the dura mater tissue provides it with a significant effect on the tissue's mechanical stiffness. Collagen is a key load-bearing structural component of soft biological tissues. ${ }^{35}$ The dense collagen architecture of the porcine dura mater is evident in Fig. 2, which shows scanning electron microscopy (SEM) images of macerated dura mater tissue. Maceration removes noncollagenic tissue components to allow for enhanced visualisation of collagen fibres. ${ }^{36}$ Work by Walsh et al (2018) conducted the first quantitative regional biochemical evaluation of the dura mater. It was observed that significant regional variation existed in a number of key structural extracellular matrix proteins including collagen I, collagen III and elastin. ${ }^{37}$ Interestingly, the authors observed significant regional differences in collagen I content, the main structural element of the extracellular matrix, ${ }^{35}$ and regions with high collagen I content generally had higher mechanical stiffness than other regions. ${ }^{37}$

Numerous investigations have found that collagen fibres within the dura mater show signs of local alignment, but this alignment occurs over short spatial distances and thus it has generally been accepted that the dura is structurally isotropic in bulk. ${ }^{27,38-40}$ 


\section{Falx cerebri and tentorium cerebelli structural composition and alignment}

The falx and tentorium are described as being composed of osteoprogenitor cells, fibroblasts and a dense network of fibrous collagen.$^{41}$ Interestingly, the falx and tentorium are ossified in a number of species, ${ }^{31}$ and while most humans possess a soft-tissue falx and tentorium, approximately $10 \%$ of the adult population exhibit partial falx ossification. ${ }^{42}$ It is currently unclear the functional role ossification of these structures plays, while hypotheses suggest it may be to provide extra protection for the brain during locomotion. ${ }^{31}$

The qualitative SEM analysis of Tatarli et al (2013) suggests that the dense collagen bundles within the falx preferentially align along parallel to the sagittal plane. ${ }^{43}$ Analysis of the nerve fibre alignment has been conducted in the tentorium cerebelli, which indicates that the fibres are oriented in the anterior to posterior direction. ${ }^{44}$

\section{Pia-arachnoid complex (PAC) structural composition and alignment}

The voluminous subarachnoid space of the PAC is occupied by cerebrospinal fluid (CSF), arachnoid trabeculae and subarachnoid vasculature. The arachnoid trabeculae consist primarily of type I collagen fibres arranged in a random three-dimensional network. ${ }^{45}$ Analysis of postmortem human subjects identified a mean volume fraction of arachnoid trabeculae of $\approx 25 \%$. ${ }^{46}$ The random fibril organisation within the trabeculae is thought to allow for stress redistribution under supraphysiological loading. ${ }^{45}$ The arachnoid trabeculae are the main load bearing component of the PAC in normal traction loading but are thought to function primarily in resisting tensile stress, as they buckle under small magnitudes of compressive load. ${ }^{47}$ Significant local variations have been observed in porcine arachnoid trabeculae and subarachnoid vasculature volume fraction, ${ }^{21}$ with regional volume fraction results ranging from $14-53 \%,{ }^{21}$ highlighting the intricate architecture of the PAC. A study on the spinal pia mater ultrastructure identified no 
preferential alignment of the pial collagen fibres in human specimens. ${ }^{48}$

\section{Mechanical characterisation of soft biological tissues: basic}

\section{concepts}

This section provides a brief introduction to concepts related to the characterisation of soft biological tissues and to the mechanical properties described in this review. The mechanical behaviour of soft biological tissues is a function of both elastic and viscous components, ${ }^{49}$ meaning that the tissues exhibit time-dependent mechanical behaviour. Soft biological tissues are capable of undergoing large tensile deformations and typically exhibit a nonlinear stiffening at high deformations. Several mathematical models have been developed to characterise the constitutive behaviour of these nonlinear, viscoelastic soft tissues. ${ }^{50}$ However, while the use of these models is desirable to fully capture the complex, time-dependent behaviour of soft

biological tissues for simulation purposes, ${ }^{51}$ the wide variety of models employed in the literature and the numerous terms in these models make comparison between experimental results difficult.

A number of elastic and viscoelastic mechanical properties pertinent to both TBI and dural graft modelling are discussed in this review. Elastic modulus values, which provide a measure of the stiffness of materials, are frequently reported for soft biological tissues in the biomechanical literature to compare the results of experimental investigations ${ }^{52,53}$ and to compare the behaviour of tissues from around the body. ${ }^{54}$ Tissues such as bone, tendon and ligament are relatively stiff biological tissues (with tensile moduli in the range of MPa and GPa), while tissues such as fat and brain tissue are among the least stiff tissues in the body (with moduli in the range of $\mathrm{kPa}$ ). ${ }^{55}$ Tensile resilience, $u_{R}$, is a measure of a tissue's ability to store and release strain energy. ${ }^{54}$ Tendons, often referred to as 'biological springs', play a key role in preserving energy during locomotion, and are an example of a tissue with high tensile resilience. ${ }^{56}$ 
Damping in a viscoelastic tissue, measured using damping loss factor ( $\tan \delta$ ), is a measure of a tissue's ability to dissipate energy associated with dynamic loading such as TBI. ${ }^{57} \mathrm{An}$ investigation of horse digital flexor muscles has shown that the flexor muscles contribute to damping the high-frequency, potentially damaging limb vibrations associated with hoof strikes on hard surfaces ${ }^{57}$ Similar protective mechanisms exist throughout the body, whereby viscous friction is utilised to dissipate the energy of impacts. ${ }^{58}$ Strain to failure, $\varepsilon$, is characterised by the percentage of deformation a tissue undergoes, relative to its original length, prior to reaching mechanical failure. Finally, tensile strength, $\sigma_{T}$, is the maximum stress a material can withstand before failing while ultimate strain is the corresponding strain. Skin tissue, which provides critical protection to vertebrates from insults such as predatorial attacks, utilises sophisticated structural features to prevent mechanical failure.$^{59}$ Both strain to failure, ultimate strain and tensile strength are important characteristics when considering the large, injurious loadings that tissues experience during dynamic events such as automotive collisions. ${ }^{60}$

\section{Review Methodology}

The cranial meninges consist of three layers; the dura mater, the arachnoid mater and the pia mater. The pia and arachnoid mater membranes, which are intimately connected via arachnoid trabeculae, are commonly referred to collectively as the pia-arachnoid complex (PAC) $)^{21}$ and will thus be described as such. From a structural perspective, the dura mater serves to anatomically compartmentalise the brain; fibrous extensions of the dura mater, such as the falx cerebri and tentorium cerebelli, partition the cranial cavity into discrete compartments. ${ }^{3}$ As these dural structures have been highlighted for their propensity to induce localised strain concentrations in brain tissue during $\mathrm{TBI},{ }^{13}$ they are discussed independent of the dura mater throughout the review. The PRISMA systematic review process was utilised to select the papers reviewed herein. ${ }^{61}$

\section{- Scopus search string:}


(TITLE-ABS-KEY (meninge* OR dura OR pia OR arachnoid OR falx OR tentorium) AND (mechanic* OR biomech*) AND (properties OR characterisation OR *elastic*) AND (LIMIT-TO (DOCTYPE , "ar" ) AND ( LIMIT-TO (LANGUAGE, "English"))

- Web of Science search string:

TOPIC: ((meninge* OR dura OR pia OR arachnoid OR falx OR tentorium) AND (mechanic* OR biomech $^{*}$ ) AND (properties OR characterisation OR *elastic $\left.{ }^{*}\right)$ )

o Search was refined by: DOCUMENT TYPES: (ARTICLE) AND LANGUAGES: (ENGLISH)

- Academic Search Complete search string:

((meninge* OR dura OR pia OR arachnoid OR falx OR tentorium) AND (mechanic* OR biomech*) AND (properties OR characterisation OR *elastic $\left.{ }^{\star}\right)$ )

o Search was completed using the default search fields. Search was limited to articles published in English.

The identified studies were then screened based on the inclusion criteria for the systematic review. Only original experimental research articles were included. Papers were excluded if they did not focus on the cranial meninges (e.g. paper focussed solely on the spinal and optic nerve meninges were excluded). Papers were also excluded if they did not conduct mechanical or structural characterisation of the meninges (e.g. studies which only focussed on finite element modelling of the meninges were excluded). The terms "falx" and "tentorium", referring to the falx cerebri and tentorium cerebelli (which are fibrous extensions of the dura mater), were included due to their influential role in determining brain strains during TBI events. ${ }^{13,23}$ Note that studies focussed on animal tissues were included in the review as significant neuroanatomical ${ }^{62}$ and neurovascular ${ }^{63}$ similarities have been noted between various mammalian species. The use of animal models in the biomechanics field is common due to a shortage of availability of human tissue surgical donations and the ethical considerations in obtaining cadaveric tissue. ${ }^{64} \mathrm{~A}$ summary of the papers 
identified and excluded in the systematic review is presented in Fig. 3.

\section{Results}

663 articles were identified based on the search criteria. The articles were screened to identify studies which focussed on mechanical evaluation of the cranial meninges. 27 of the 663 articles were ultimately included in the review. The reasons for article exclusion are as described in Fig. 3 (A). 4 additional articles, which were not identified in the systematic review but were of relevance to meningeal mechanics, were added to the review (see Fig. $3(\mathrm{~A})$ ). Of the 31 articles evaluated in the review, 30 of the articles conducted experimentation on cadaveric material while just 1 article conducted experimentation on in vivo tissue. The articles were then screened and evaluated based on a number of mechanical testing parameters (see supplementary tables $\mathrm{S1}$ S6 in the supplementary file for detailed analysis data). As demonstrated in Fig. 3 (B), it was identified that the majority of identified studies focused on dura mater mechanics $(n=19)$, followed by the PAC $(n=5)$ and indentation analyses of whole meninges $(n=3)$. No peer-reviewed studies were identified examining the mechanics of the falx and tentorium tissues. Of the studies which focussed on dura mater mechanics, uniaxial tension was the most common modality of testing $(n=14)$, followed by membrane inflation $(n=2)$, free vibration analysis $(n=1)$, biaxial flexure $(n=1)$ and planar biaxial testing $(n=1)$, see Fig. $3(C) .80 \%$ of the studies focussed on dura mater mechanics tested human tissue (see Fig. 4 (B)), while the other $20 \%$ of studies utilised porcine, rat or monkey models. All of the 5 PAC mechanics studies conducted testing on either bovine $(80 \%)$ or rat $(20 \%)$ models (see Fig. $4(\mathrm{C}))$. The modalities of testing employed in the 5 PAC publications were uniaxial tensile testing $(n=2)$, atomic force microscopy $(A F M)(n=1)$, shear testing $(n=1)$ and normal traction testing $(n=1)$, see Fig. $3(D)$. In contrast to the dura mater

investigations, none of the 5 PAC investigations were focussed on human tissue, with bovine tissue being evaluated in the majority (80\%) of investigations (see Fig. $4(\mathrm{C})$ ). 
Fig. 5 (B) provides an analysis of the thickness measurement techniques utilised in the identified studies. Almost $70 \%$ of the studies utilised contact-based measurement such as micrometers, dial indicators and digital callipers while $\approx 30 \%$ of the studies utilised noncontact methods such as noncontact photogrammetry and cast and scanning. Fig. 5 (C) provides an analysis of various test parameters observed in the identified studies. It was observed that $59 \%$ of the studies conducted tissue preconditioning, only $45 \%$ of studies analysed local sample deformation during sample characterisation, $62 \%$ of the studies did not submerge samples in a saline bath during characterisation to maintain in vivo hydration conditions, while just $19 \%$ of the studies tested the meningeal samples at physiological temperature.

The following sections review the different meningeal regions in relation to a number of important mechanical characteristics. Capturing the nonlinear behaviour of meningeal tissues is an important consideration for constitutive modelling purposes. ${ }^{65}$ However, for the purposes of simplified quantitative analysis of meningeal tissue characteristics, linear elastic moduli values from the identified studies are evaluated in Fig. $4(\mathrm{~A})$. The elastic moduli in Fig. $4(\mathrm{~A})$ were calculated from the linear region of the stress strain curve (i.e. after the initial 'toe-region') and were reported by the original study authors. Note that the modulus value assigned to the dura, falx and tentorium in the majority of FE models (31.5 MPa based on the work of Galford et al (1970) on human dura mater tissue ${ }^{66}$ ) is highlighted for comparison purposes, see Fig. 4 (A). Given the importance of geometrical property quantification for the calculation of tensile stresses and for modelling applications, the reported geometrical properties in the identified studies are also reviewed.

\section{Dura mater mechanics}

\section{Overview of dura mater mechanical behaviour}

The dura mater is a notably stiff soft biological tissue with significant nonlinear and viscoelastic 
behaviour. ${ }^{67,68}$ Many modalities of mechanical analysis have been conducted to investigate dura mater mechanics, as shown in Fig. 3 (B). As discussed in section 1.2.1, the collagenic alignment of the dura mater is highly variable. Thus, it is not surprising that significant inter and intra-subject variability has been observed for the dura mater. ${ }^{37,69}$ The large variability of dural mechanics is also evident in the relatively large standard deviations of the data presented in Fig. $4(\mathrm{~A})$. The wide variety of species tested in dura mater mechanics studies is demonstrated in Fig. 4 (B). However, elastic moduli have only been reported for human and porcine tissues, see Fig. 4 (A). Comparing the literature values for porcine and human dura, it appears that porcine dura has smaller elastic moduli values than the human dura mater tissues. Comparing the moduli values with that of the value conventionally assigned to the dura mater in FE models which utilise a linear elastic model for the dura (as opposed to a nonlinear material model which is utilised in many recent FE models), the linear elastic model with a value of $31.5 \mathrm{MPa}$ conventionally assigned to dura mater in FE models appears to be an underestimation of dural stiffness, with the majority of studies on human dura reporting higher mean moduli than $31.5 \mathrm{MPa}$ (see Fig. 4 (A)).

\section{Mechanical isotropy analyses of dura mater}

A study by Sacks et al (1998) on human cranial dura mater identified that the local collagenic alignment of the dura mater had a significant effect on the tissue's local mechanical properties. ${ }^{26}$ It was found that test samples with a collagen alignment parallel to the direction of uniaxial tensile testing were both stiffer (exhibiting a higher elastic modulus) and stronger (higher ultimate tensile strength) than samples with fibres aligned perpendicular to the direction of uniaxial tension. ${ }^{26}$ Thus, they concluded that while the tissue did appear to exhibit mechanical anisotropy in bulk, small constituent regions of the tissue exhibited significant structural and mechanical anisotropy. Numerous investigations on the anisotropy of bulk dura mater tissue have confirmed that the tissue exhibits bulk isotropic mechanical behaviour. ${ }^{37,38,69}$ 


\section{Strain rate-dependency of dura mater}

There is a dearth of experimental data on the strain rate-dependence of cranial dura mater. Previous work utilised dynamic indentation to estimate the damping ratio of the dura mater and brain over a wide range of frequencies $(0.01-100 \mathrm{~Hz}) .{ }^{17}$ The damping ratio of a tissue provides an indication of how efficient a tissue is at dissipating energy. It may thus provide an indication of how tissues such as the meninges provide protection during TBI. ${ }^{17}$ It was identified that the dura mater tissue exhibits less damping capacity at higher, TBI-relevant frequencies, suggesting that the dura is not effective at providing protection to the brain at higher frequencies.

However, no studies were identified on the rate-dependency of other mechanical parameters for the cranial dura such as stiffness, ultimate strain and tensile strength.

\section{Regional dependence of dura mater stiffness}

A number of the identified studies have investigated the regional dependency of the dura mater and have found that the native dura mater tissue does not appear to display regional anisotropy. ${ }^{37}$, 69,70

\section{Age-dependency of dura mater}

Van Noort et al (1981) studied the effect of donor age on dura mater tensile strength utilising uniaxial tensile testing with donors ranging in age from 24 to 88 years. They identified a highly significant $(r=-0.79 ; p<0.001)$ linear decrease in the tensile strength as a function of donor age. ${ }^{71}$ Similar to the analysis of Van Noort et al (1981), Zwirner et al (2019) evaluated the influence of dura mater tissue donor age utilising uniaxial tensile testing in subjects ranging from 2 to 94 years. They identified a relatively weak negative correlation between elastic modulus $(r=-0.283 ; p=$ $0.002)$ and tensile strength $(r=-0.299 ; p=0.001)$ to the donor age at death. ${ }^{70}$ 
In relation to dural mechanical changes during development, an investigation by Kriewall et al (1983) on foetal dura, identified a positive correlation between fetal age in weeks and dural stiffness. ${ }^{30}$ Furthermore, a stiffening of intracranial tissues when comparing immature and mature rat tissues has also been observed. ${ }^{72}$ Thus, dura mater appears to stiffen in the early stages of mammalian development.

\section{Geometrical analyses}

As demonstrated in Fig. $5(\mathrm{~A})$, many studies have reported on dura mater geometrical properties. A large range of values has been reported for dura thickness (with mean thickness values ranging from $0.35 \mathrm{~mm}^{26}$ up to $1.11 \mathrm{~mm}^{73}$ ), suggesting significant inter-subject variability.

\section{Falx cerebri and tentorium cerebelli mechanics}

No peer-reviewed investigations were identified focusing on the mechanical properties of the falx and tentorium in this systematic review. To the best of the authors' knowledge, the only study investigating the material properties of the falx and tentorium is the non-peer reviewed work of Golman et al (2013). The authors conducted indentation and uniaxial tensile tests of the falx and tentorium tissues. ${ }^{74}$ Interestingly, regional analysis of the medial-lateral stiffness identified that the falx had a decreased stiffness at increased distances from the skull. Uniaxial tensile tests were conducted at strain rates ranging from quasi-static $(0.001 / \mathrm{s})$ to TBI-mimetic strain rates (10 /s) and identified a significant rate-dependent behaviour in the tissues (see supplementary table S4). However, peer-reviewed experimental studies are required to provide any conclusions on falx and tentorium mechanics.

\section{Geometrical analyses}

The non-peer reviewed work of Golman et al (2013) identified a mean falx thickness of $0.45 \pm$ 
0.16 (s.d.) $\mathrm{mm}$ and tentorium thickness of $0.36 \pm 0.15$ (s.d.) mm. As demonstrated in Fig. 5 (A), these values are in the same range as values reported for native dura mater tissue. Previous analysis, while not identified in this review, conducted analysis of in vivo falx and tentorium geometries from computed tomography images. ${ }^{75}$ They reported the mean length of the falx's projection into the longitudinal fissure as $41.8 \pm 5.9$ (s.d.) $\mathrm{mm}$ and the mean length of the tentorium's projection into the transverse fissure as $99.64 \pm 4.79$ (s.d.) $\mathrm{mm} .{ }^{75}$ However, falx and tentorium thicknesses were not reported.

\section{Pia-arachnoid complex (PAC) mechanics}

\section{Overview of PAC mechanical behaviour}

The pia and arachnoid membranes are intimately mechanically tethered to one another. ${ }^{76}$ Thus, as no research group has described an adequate separation protocol of the membranes, it is assumed that all investigations of either the pia or arachnoid membranes are indeed investigations of the PAC.

Uniaxial tensile testing conducted by Aimedieu and Grebe (2004), reported on the elastic properties of the bovine cranial PAC. The authors observed a significant nonlinear stress-strain response and the ultimate strain of the tissue was 0.19 . A limitation of this investigation is the lack of specimen geometrical analysis, and thus tensile stresses and accordingly elastic moduli were not calculated.

The comprehensive work of Jin et al includes a series of investigations on PAC mechanics for TBI computational modelling purposes. ${ }^{76-78}$ The first of these analyses, similar to the work of Aimedieu and Grebe (2004), conducted tensile testing of the bovine PAC. Samples were tested in two perpendicular testing directions to evaluate potential tissue anisotropy. The study found that the PAC tissue exhibited a nonlinear mechanical response and was transversely isotropic. Direct comparison between the tensile testing results of Aimedieu and Grebe (2004) and Jin et al 
(2006) is not possible as thickness values were not calculated by the former. ${ }^{79}$ However, based on the load-deformation and specimen size data reported in the publication by Aimedieu and Grebe (2004), Jin et al calculated the elastic moduli values of the results of Aimedieu and Grebe based on the representative pia thickness observed in the investigations of Jin et al. ${ }^{76}$ With a representative mean thickness of $23.6 \mu \mathrm{m}$, it was established that the mean elastic modulus value was $9.43 \mathrm{MPa}$. This is approximately in agreement with the quasistatic moduli results of Jin et al of $6.75 \mathrm{MPa} .{ }^{76}$ Slight variations were also observable in the ultimate stress and strain values, likely a result of varying preloading protocols. ${ }^{76}$

In another investigation by Jin et al, normal traction loading of the PAC was conducted. ${ }^{77}$ Unlike the tensile testing analysis, which allowed for evaluation of the load-bearing capacity of the arachnoid and pia membranes, the normal traction loading evaluated the load-bearing properties of the arachnoid trabeculae. PAC samples were glued to polyethylene blocks on both the pia and arachnoid surfaces. Interestingly, the tests revealed that the PAC had essentially linear stressstrain behaviour under normal traction loading (with mean quasi-static moduli of $61 \mathrm{kPa}$ ). This is in contrast to the nonlinear behaviour commonly associated with soft biological tissues,,$^{80}$ and to the hyperelastic behaviour of the tissue under in-plane tension. ${ }^{76} \mathrm{It}$ is unclear why the load-bearing trabeculae exhibit this stress-strain response ${ }^{77}$ but it is likely due to unique collagen alignment in the structures. In their final investigation of PAC mechanics, shear loading of the PAC identified mean shear moduli of $11.73 \mathrm{kPa}$. Note that both the traction and shear loading moduli of the PAC are lower than the moduli observed in tensile testing of the PAC, likely due to the contribution of the dense collagen network within the pia and arachnoid membranes in tensile loading. ${ }^{81}$

\section{Strain rate-dependency of PAC}

In contrast to the dearth of data on the rate-dependency of the cranial dura mater, the ratedependency of the PAC has been evaluated in a number of the aforementioned investigations by 
Jin et al. In all three investigations and associated loading conditions, the effects of a range of TBI-relevant strain rates (from quasi-static up to 100/s) identified significant rate-dependent behaviour in all three loading modalities (see supplementary table S6 for details). Therefore, it can be concluded that the PAC tissue has strong rate-dependence in multiple modalities of loading. This rate-dependency is of particular interest for TBI modelling applications.

\section{Regional dependence of PAC stiffness}

In all 3 investigations by Jin et al described in the sections above, no regional dependence was identified in bulk PAC mechanics. ${ }^{76-78}$ However, the work of Fabris et al (2019) identified significant local variation in PAC mechanical properties. Utilising a combined approach of AFM and immunofluorescent imaging, the authors studied the local structure-function relationship of the PAC. Statistically significant differences between the elastic moduli distributions between vascularised and non-vascularised regions, again highlighting how this tissue can cause large variations in regional load propagation characteristics. They observed mean elastic moduli, $\mathrm{E}=$ $1595 \pm 55 \mathrm{~Pa}$ (standard error) in non-vascularised regions, which as they highlight is comparatively less than the studies which have evaluated whole tissue PAC mechanics described above. They attribute this difference to the modality of testing employed (elastic moduli in the order of $\mathrm{kPa}$ are typical for extracellular matrix level mechanics) and the use of a rat model. ${ }^{34}$ Interestingly, the authors highlight that the values they observed are approximately 2- to 10 -fold higher than the values reported for AFM analysis of parenchymal tissue.

Thus, similar to the cranial dura, the PAC appears to exhibit isotropic properties in bulk but has considerable local anisotropy at smaller scales due to structural heterogeneity.

\section{Age dependency of PAC mechanics}

No studies investigating the age-dependency of the PAC were identified in this review. 


\section{Geometrical analyses}

A major limitation of measuring the geometrical properties of the PAC is the post-mortem propensity for CSF to evacuate the subarachnoid space ${ }^{21}$ Measurements of the depressed PAC indicate the membrane has average thickness of $23.6 \pm 5.8$ (s.d.) $\mu \mathrm{m}$. Note that this value was obtained without inflation of the subarachnoid space. ${ }^{76}$

While not identified in this review, previous analysis of porcine heads employed constant volume injection of saline to reinflate the subarachnoid space to physiological levels. ${ }^{21}$ However, it was noted that it is impossible to determine if the brains were inflated to representative in vivo conditions. Utilising optical coherence tomography, the arachnoid membrane thickness was identified as $27.06 \pm 5.57$ (s.d.) $\mu \mathrm{m}$, while the subarachnoid space thickness was identified as $287.92 \pm 151.12$ (s.d.) $\mu \mathrm{m}$. The mean subarachnoid vessel diameter was $183.74 \pm 83.32$ (s.d.) $\mu \mathrm{m}$ while measurements of the significantly smaller arachnoid trabeculae were not attained. The authors did not measure pia mater thickness, presumably as the membrane was indistinguishable from the underlying brain tissue. Another study, again not identified in the systematic review, utilised OCT imaging intra-operatively to analyse the in vivo human PAC. ${ }^{82}$ They identified a mean subarachnoid space thickness of $570 \mu \mathrm{m}$ and a mean arachnoid membrane thickness of $74 \mu \mathrm{m}$. Interestingly, these values are both larger than the dimensions identified in the ex vivo porcine subjects.

\section{Discussion}

In this systematic review we identified and screened 663 original research articles and reviewed 27 studies which have evaluated the mechanical properties of the cranial meninges. We provide an analysis of the mechanical properties reported in these publications to aid in both biofidelic constitutive modelling of meningeal tissues in TBI models and the design of dural graft materials. 
Furthermore, based on the experimental limitations observed in the reviewed literature and the numerous open questions identified regarding meningeal mechanics, the following sections provide recommendations to researchers on how meningeal mechanics experiments can be conducted to enable standardised testing methodologies. Finally, suggestions on future directions for meningeal mechanics studies to address current knowledge gaps are provided.

The mechanical properties of various soft biological tissues are as presented in the multidimensional radar plots shown in Fig. 6 (see supplementary table S7 for data sources). The tissues are compared over a number of relevant mechanical characteristics utilising a quantitative performance mapping system. ${ }^{54}$ As demonstrated in Fig. 6, the dura mater exhibits less damping capacity than both the brain and scalp. The limited damping capacity of the dura potentially suggests the dura mater is poor at dissipating the harmful levels of energy associated with TBI. ${ }^{83}$ The dura mater demonstrates increased stiffness and strength compared to the other soft biological tissues (Fig. 6). The large stiffness gradient between the meninges and the underlying brain tissue has important implications for the mechanics of $\mathrm{TB}^{13,84}$, and therefore inaccurate representation of the tissues in computational models of TBI can have a significant impact on model findings. ${ }^{19,65}$ Currently, the dura, falx and tentorium are represented with a linear elastic model in FE models with a value of $31.5 \mathrm{MPa} .{ }^{13}$ However, as identified in this systematic review, this appears to be an underestimation of dura mater stiffness (Fig. 4 (A)), while there is a significant dearth of properties for both the falx, tentorium and the PAC (Fig. 4 (A)). The following sections provides suggestions for future directions of meningeal mechanics investigations.

\section{Experimental limitations in evaluating meningeal mechanics}

This section highlights the identified limitations in current experimental evaluation of meningeal mechanics along with suggestions on how these limitations may be addressed. 


\section{Replicating in vivo hydration and temperature during experimentation}

As illustrated in Fig. 5 (C), 62\% of investigations characterised samples without tissue submersion in a physiologically-relevant solution. It has been noted that the dura mater has a large surface area to volume ratio and dehydrates rapidly. ${ }^{66}$ Previously, a study on the effects of dehydration on ligament tissue demonstrated that dehydration causes up to a $50 \%$ reduction in storage compliance. ${ }^{85}$ The capacity for dehydrated tissues to rehydrate is dependent on the initial extent of dehydration, and complete rehydration is often not possible. ${ }^{86}$ It was also noted that $81 \%$ of the identified studies did not maintain physiological temperature during testing, see Fig. $5(\mathrm{C})$. Testing soft biological tissues below physiological temperature can significantly alter tissue mechanics. ${ }^{87}$ Ideally, test samples should be fully submerged in a physiologically-relevant solution at $37^{\circ} \mathrm{C}$ throughout testing. ${ }^{88}$

\section{The effects of removing tissue from the in vivo environment}

In situ characterisation of biological tissues is desirable as the tissue can be evaluated in its natural environment, thus negating many of the issues associated with ex vivo characterisation. The natural environment of the meninges entails an intact CSF environment ${ }^{72}$ and in situ strain on the dura mater induced by its connection to the skull. ${ }^{89}$ It has been noted that the CSF drains from the subarachnoid space following post-mortem animal decapitation ${ }^{21}$ and this likely results in alterations in PAC mechanics. Many studies have indeed tested in situ meningeal tissue utilising indentation-type loading, ${ }^{17,} 18,72$ and at least for the underlying brain tissue, significant differences were observed between in situ, in vitro and in vivo testing. ${ }^{18}$ Another study evaluating the in situ, in vitro and in vivo mechanics of the rat meninges-CSF-brain complex identified that while the elastic properties of the complex did not significantly differ when comparing in vivo and in situ results, there were significant post-mortem changes observable in the viscoelastic properties of the complex. ${ }^{72}$ The differences between the various testing conditions in both studies 
were attributed to the changing boundary conditions between in vitro and in situ testing ${ }^{18}$ and due to post-mortem tissue alterations associated with ex vivo testing. ${ }^{72}$

While it is desirable to test meningeal tissue in vivo, studying the behaviour of the meninges in a variety of loading deformations is desirable to replicate the multi-dimensional nature of TBI. Furthermore, tensile properties are of key interest for dural graft design. Traditional mechanical testing setups require specific specimen geometries, ${ }^{88}$ and thus tissue excision from the in situ environment for ex vivo testing is required.

Regarding the degradation of soft biological tissues following tissue donor death and the associated alterations in mechanical integrity, it is recommended that tissues are either stored in a physiologically-relevant solution at $4^{\circ} \mathrm{C}$ and tested within 24 hours of donor death ${ }^{88}$ or are cryopreserved in cryoprotectant media at $-80^{\circ} \mathrm{C}$ to mitigate structural and mechanical degradation during longer term storage. ${ }^{90,91}$

\section{Measurement of sample geometries for sample stress evaluation}

As demonstrated in Fig. 5 (B), a wide variety of equipment to measure test sample width and thickness has been identified in this systematic review. Soft biological tissues undergo significant deformation at low loads and are thus susceptible to erroneous sample cross-sectional area measurement utilising handheld measuring tools such as callipers and micrometers. Inaccurate assessment of sample cross-sectional area can result in significant errors in the calculation of experimental tensile stresses and can thus influence experimentally-derived tissue mechanical properties. ${ }^{92}$ Consequently, future studies should focus on the use of noncontact photogrammetry methods ${ }^{37}$ or laser scanning methods ${ }^{93,94}$ to determine sample cross-sectional area.

\section{Preconditioning of test samples}

Here we identify that $41 \%$ of the identified studies did not conduct sample preconditioning, when 
it is appropriate to do so (i.e. for tensile and inflation testing). Preconditioning is a process by which test samples are subjected to 2-10 loading and unloading cycles at low magnitude of strain (1 $-10 \%)$ prior to sample characterisation to ensure a repeatable mechanical response. ${ }^{88}$ Preconditioning is characterised by stress softening of samples with successive loading cycles. Samples are said to be 'preconditioned' once the stress softening effects and the test samples

demonstrate a repeatable response ${ }^{95}$ Given the stiff nature of meningeal tissue when compared to other soft biological tissues (see Fig. 6), care should be taken to prevent microstructural damage to meningeal tissue during preconditioning and thus influencing test results. ${ }^{96}$ Thus, we recommend preconditioning meningeal samples to 10 cycles of $3 \%$ strain.

\section{Tracking of local sample deformation}

Digital image correlation (DIC) is an image-based technique that can track test sample displacement based on the deformation of a pattern on the sample surface throughout a test. Use of DIC allows for elimination of any potential error associated with sample slippage from the testing clamps. Measuring sample displacement from the grips displacement alone may result in a significant overestimation of the stretch the sample has been exposed to, should sample slippage occur. ${ }^{97} \mathrm{DIC}$ also eliminates any potential error associated with stress concentrations at the grip-sample interface ${ }^{98}$ or due to irregular test sample shapes. ${ }^{99}$ Typically, to negate the erroneous effects of stress concentrations at the grip-sample interface, uniaxial test samples are tested in pure tension with a length to width ratio of $5: 1 .^{88}$ However, DIC allows for complete elimination of both grip concentrations and irregular sample geometries by characterising local tissue deformation ${ }^{98}$ and is therefore recommended for meningeal mechanics investigations when possible.

\section{PAC sample isolation}


The extremely minute geometries of the PAC, see section 3.3.5, presents significant experimental challenges. It has been noted by Jin et al (2006), that it is almost impossible to separate the arachnoid and pia mater layers, which are tightly fused together by arachnoidal trabeculae. Consequently, the tissue structure makes ex vivo separation of the membranes impossible without excessive damage. ${ }^{76}$ Furthermore, detaching the pial membrane from the brain cortex without inducing mechanical damage is a significant challenge. Many methods have been described to isolate the PAC. In the work of Aimedieu and Grebe (2004), PAC tissue was isolated by placing the PAC surface on a paper template, scraping away the viscous brain tissue, and then placing the paper template with the attached PAC in a saline solution. Once the paper became weakened by the water, the fragile PAC was separated from the deformable support template. ${ }^{79}$ In a similar fashion, Jin et al (2006) utilised a polyethylene sheet to isolate PAC with 5 to $10 \mathrm{~mm}$ of underlying brain. The brain tissue was then carefully removed from the pial junction. ${ }^{76}$ In the work of Fabris et al (2019), a dissecting microscope and a fine tip, high precision tweezers were used to remove PAC fragments from a rat brain.

It is unclear if the aforementioned methods induce any mechanical or structural damage to the PAC tissue prior to characterisation. Novel isolation methods that do not require expert levels of skill and precision are desirable to ensure consistent tissue isolation which may address the dearth of human PAC data and improve TBI modelling biofidelity.

\section{PAC sample gripping}

It has been noted that particular caution should be exercised during the preparation of fragile tissues, such as brain tissue,${ }^{64}$ for mechanical characterisation. The exceedingly small geometries of the PAC (see section 3.3.5) presents unique challenges for studies wishing to evaluate PAC mechanics in torsion and normal tension, both of which are relevant for evaluating multiaxial TBI loading. 
In the work of Jin et al (2007) and (2011), cyanoacrylate glue was used to adhere the PAC membranes to test fixtures for measurement of shear and normal traction mechanical response. As the authors discuss, there are two concerns regarding the use of glue for specimen adhesion to test fixtures. Firstly, any penetration of this glue into the PAC samples would cause a significant artificial stiffening of test samples. However, it has been noted previously that the fibroblast cells of the arachnoid form a somewhat permeable layer. ${ }^{100}$ Thus, it is unclear if the glue could permeate through this layer and alter the mechanical and morphological features of the PAC. Jin et al (2009) investigated the extent of glue penetration using a combination of histological methods and analysis under a light microscope. They observed a nonsignificant difference in thickness of glued and native tissues, suggesting the glue did not induce morphological changes in PAC specimens, at least under an optical microscope at $400 x$ magnification. ${ }^{81}$

The AFM work of Fabris et al (2019) investigated the effects of adhesives on the PAC mechanical response and noted some differences between adhesive coated samples and native controls. Future investigations of PAC shear and normal traction properties should confirm that glue does not diffuse into the PAC membrane through more advanced analysis techniques such as energy dispersive X-ray (EDX), a powerful elemental analysis technique that reveals the presence of elements in various locations of a specimen. ${ }^{101}$

\section{Open questions and future directions}

\section{The mechanical response of the falx and tentorium}

A major finding of this systematic review is the lack of mechanical properties of the falx cerebri and tentorium cerebelli. The early modelling work of $\mathrm{Li}$ et al (2007) utilised a two-dimensional FE model to explore the influence of both a flexible and rigid falx on brain strains. The presence of the falx and tentorium resulted in a decrease in brain tissue strains in peripheral brain regions, 
with an increase in strain within deep brain structures, such as the corpus callosum. Interestingly, the authors also observed an increase in strain within the brainstem, which they hypothesised to be a result of the presence of the tentorium. ${ }^{102}$

The authors speculate that the mechanism of this localised strain observed within the corpus callosum and brainstem is related to the stiff falx and tentorium consolidating movement of the cerebral hemispheres and cerebellum, respectively. The non-peer reviewed work of Golman et al (2013) suggests that the stiffness of the dural structures exceeds the elastic modulus that is currently assigned to them ${ }^{74}$, see Fig. 4 (A). Thus, there is a pressing need for constitutive mechanical data on the falx and tentorium for FE modelling applications.

\section{Species-dependence of meningeal mechanics}

Significant anatomical similarities exist in the neuroanatomy of various species. ${ }^{62}$ Porcine tissue, in particular, is used frequently in neuroscience applications. ${ }^{103}$ Studies on the speciesdependence of brain tissue mechanics have thus far been inconclusive in elucidating any speciesdependent differences in brain tissue mechanics. ${ }^{64}$ To date, no evaluation has been conducted on meningeal tissue species-dependence. The few studies which have evaluated the PAC and the in situ whole meninges have all been conducted on animal models, see Fig. 4 (C). As characterisation of the meninges is conducted for the understanding of human health and disease, understanding the species-dependent nature of the PAC and whole meninges is a key consideration.

\section{Strain rate dependency of meningeal tissues}

Automotive crashes are the number one cause of injury-related death in the United States. ${ }^{104} \mathrm{FE}$ analyses of injury data obtained from controlled automotive crash tests with crash dummy occupants have provided invaluable data on the tissue-level strains which occur in various impact 
scenarios. ${ }^{16} \mathrm{~A}$ recent $\mathrm{FE}$ analysis on the strain rates observable in the dura mater during various impact scenarios found that strain rates of up to 103 /s were possible in the dura mater during an impact which corresponded to a mild TBI. ${ }^{105}$

Much work has been conducted on the strain rate behaviour of brain tissue and many studies have demonstrated its strong strain rate dependency.${ }^{17,106,107}$ This review identified a number of studies suggesting that the PAC has significant rate-dependent mechanical behaviour, which has important implications for TBI modelling. However, the review identified a dearth of data on the rate-dependent behaviour of the dura mater, falx and tentorium. Previously, a study on bovine spinal dura mater tissues identified no significant difference between tissues tested at strain rates ranging from 0.01 to $1 / \mathrm{s} .{ }^{108}$ However, it should be noted that while the strain rates utilised in this study represent a 100-fold difference between the minimum and maximum rate, these strain rates are still relatively low when compared to TBI-relevant strains. Work by Monea et al (2014) examined the strain rate dependency of the bridging vein-superior sagittal sinus complex. They found no significant strain-dependent behaviour in the veins with strain rates ranging from $\approx 2$ 200/s. ${ }^{109}$ However, similar investigations are required for cranial dura mater, the falx and tentorium to evaluate these tissues' strain rate dependency.

\section{Replicating injury-level strain magnitudes during tissue}

\section{characterisation}

A plethora of both computational ${ }^{13,110,111}$ and experimental ${ }^{112-114}$ studies have explored the magnitude of strain that various regions of the brain are exposed to during TBI. However, little attention has been given to the magnitude of strain that the various layers of the meninges are exposed to during TBI-related loading. Identifying the strains experienced by the meninges during TBI loading will enable improved selection of constitutive models to model injury-level impacts. Techniques such as nano-indentation and AFM based indentation can be limited to moderate the 
levels of strains applied which may be insufficient in modelling even mild TBI. ${ }^{115-117}$ Thus, it is recommended that computational modelling investigations report their meningeal strain magnitude and strain rate results. Additionally, experimental characterisation researchers should also be mindful of the magnitude of strain their prospective characterisation technique is capable of achieving. Furthermore, due to the limited magnitudes of strain achievable in experimental techniques which utilise indentation-type loading, studies should report the magnitude of strain achieved during experimentation (which ideally is verified utilising finite element analysis ${ }^{115}$ ) such that the applicability of the experimental results to TBI modelling can be considered.

\section{Age-dependence and the effects of pathology on meningeal}

\section{mechanics}

Both children ${ }^{118}$ and the elderly ${ }^{119}$ have higher rates of TBI when compared to adults. Therefore, identifying age-dependent mechanical behaviour is important for demographic-specific modelling efforts. Investigations have begun to investigate differing demographics including models evaluating acute subdural haematoma risk in the elderly population ${ }^{120}$ and similarly, modelling of children's falls in playgrounds. ${ }^{121}$ As discussed in section 3.1.5, few studies have evaluated the age-dependency of the dura mater, while no studies have evaluated the age-dependency of the isolated PAC, falx and tentorium.

A study evaluating the effect of maturation on intracranial mechanical properties in rats utilised indentation testing on the intact meninges-CSF-brain complex. ${ }^{72}$ The authors observed a significant increase in both modulus of elasticity and indentation modulus of the tissues when comparing immature rat groups (aged 1-2 days and 10-days) to more mature rats (aged 56-70 days and 180 days). While rat dura mater tissue is known to have larger mechanical stiffness than the CNS tissues it surrounds, ${ }^{40}$ the authors conclude that the various layers of the brain-meninges complex make proportionally similar contributions to the observed mechanical properties. ${ }^{72}$ This 
is in contrast to the results of MacManus et al (2017), in which indentation analysis was conducted on porcine tissue, which concluded that the dura mater absorbed a significant proportion of the stresses and strains during dynamic indentation of the intact meninges and underlying brain. However, the differing conclusions of both authors may be explained by the potentially significant interspecies differences in the porcine and rat neuroanatomy. While the rat dura mater has a thickness of $13 \mu \mathrm{m},{ }^{72}$ the porcine dura mater has a thickness in the region of $0.7 \mathrm{~mm}$ to $1 \mathrm{~mm} .{ }^{37}$ Therefore, while it is difficult to ascertain the proportional contribution of the dura mater to the observed age-dependent mechanical variations of the rat tissue observed by Shulyakov et al (2011), the results follow a similar trend to Kriewall et al (1983) of increasing mechanical stiffness of dura mater with increasing age during development.

In relation to elderly subject tissue properties, FE modelling studies investigating TBI in elderly cohorts have accounted for the brain atrophy associated with aging and found that atrophied

brains have a propensity to exacerbate the risk of acute subdural haematoma. ${ }^{122}$ It is currently unclear if the age-related decrease in meningeal tissue stiffness and strength in elderly cohorts influences TBI modelling results. Further, the effects of meningeal pathology on tissue mechanics has not yet been explored and thus consideration of the effect of pathology on TBI model choice and dural graft design warrants further investigation.

\section{Residual strains of meningeal tissue}

In vivo, many biological tissues such as ligament and vascular tissues, are under residual stress and strain. When the tissues are excised from the body the in vivo stress and strain is removed, yielding a relatively stress-free configuration and resulting in retraction of the excised tissue. ${ }^{11}$ In vivo, the falx and tentorium are 'taut' to restrain brain motion. ${ }^{41}$ Similarly, a study on the dura mater of rats found that large tensile strains exist in immature rats dura mater (4.96 $\pm 1.54 \%$ s.d.) and moderate strains in mature rats $(0.39 \pm 0.13 \%$ s.d. $){ }^{89}$ To improve the accuracy of 
tensile testing analyses of ex vivo biological tissues, it is encouraged to apply a 'preload' to tissues to eliminate sample slack and more closely replicate in vivo stress and strain conditions prior to tissue characterisation. ${ }^{88}$

Furthermore, studies simulating the in vivo behaviour of arterial tissue have shown that inclusion of biofidelic prestresses on tissues improve the predictive capacity of these models. ${ }^{123}$ Similarly, Golman et al (2013) demonstrate that utilising inverse optimisation analysis of their indentation test analyses, a pretension value of $0.1 \%$ assigned to the falx cerebri in a computational model resulted in the best match between experimental data and computational modelling results. Similar analysis on a whole-head FE model identified that a falx pretension value of $6.52 \%$ produced the most biofidelic head model. ${ }^{124}$ Therefore, consideration should be given during meningeal tissue characterisation to the importance of pre-stressing tissues.

\section{Structural characterisation of the meninges}

Characterisation of the structural architecture of the meninges holds significant promise in addressing many of the currently open questions regarding meningeal mechanics and improving meningeal modelling efforts. Study of the structural architecture of the meninges has been conducted using both small angle light scattering (SALS) ${ }^{26,27}$ and SEM. ${ }^{25}$ However, SEM is limited to surface layer analysis and SALS is limited to relatively thin specimens. Thicker tissues (typically greater than $800 \mu \mathrm{m}$ ), such as dura mater tissue, are likely to produce inaccurate results. ${ }^{125}$ Therefore, imaging modalities such as second harmonic generation (SHG) are suggested. SHG allows for layer-specific microstructural characterisation of collagenic architecture ${ }^{126}$ and has been used extensively in other soft biological tissues such as tendon ${ }^{127}$ and, thus, may enable enhanced structural characterisation of the meninges.

\section{Publishing of experimental datasets}


To enable easier application of mathematical models which enable constitutive modelling of the nonlinear behaviour of the meninges and consequently to allow for easier comparisons between experimental studies, we recommend that, when possible, investigators in the field of biological tissue mechanics publish their complete experimental datasets. These datasets should contain all relevant experimental data such as, but not limited to, the test sample geometries, the experimentally measured forces and test sample deformations. This will overcome many of the limitations associated with relying on elastic modulus values to compare between literature. ${ }^{53,128}$

\section{Conclusion}

We have provided the first systematic review of meningeal mechanics, identifying 27 original research articles. Several groups have conducted mechanical characterisation of the dura mater with a wide range of employed experimental techniques, exhibiting a wide range of reported results. In contrast, there is a dearth of literature on the PAC, particularly on human subjects, while no peer-reviewed literature exists on the mechanics of the falx and tentorium membranes. We believe the suggestions provided herein may serve as a guide to future meningeal mechanics researchers to enable improved standardisation of test methodologies and easier comparison between study results. Further, the recommendations for future work will serve a starting point to improve the efficacy of dural substitutes and for improved FE modelling of TBI.

\section{Author Disclosure Statement}

No competing financial interests exist. 



\section{References}

1. Marieb, E.N. and Hoehn, K. (2007). Human anatomy \& physiology. Pearson education.

2. Rua, R. and McGavern, D.B. (2018). Advances in Meningeal Immunity. Trends in molecular medicine 24, 542-559.

3. Decimo, I., Fumagalli, G., Berton, V., Krampera, M. and Bifari, F. (2012). Meninges: from protective membrane to stem cell niche. American Journal of Stem Cells 1, 92-105.

4. Herisson, F., Frodermann, V., Courties, G., Rohde, D., Sun, Y., Vandoorne, K., Wojtkiewicz, G.R., Masson, G.S., Vinegoni, C., Kim, J., Kim, D.-E., Weissleder, R., Swirski, F.K., Moskowitz, M.A. and Nahrendorf, M. (2018). Direct vascular channels connect skull bone marrow and the brain surface enabling myeloid cell migration. Nature Neuroscience 21, 1209-1217.

5. Russo, M.V., Latour, L.L. and McGavern, D.B. (2018). Distinct myeloid cell subsets promote meningeal remodeling and vascular repair after mild traumatic brain injury. Nature Immunology $19,442-452$.

6. Coles, J.A., Myburgh, E., Brewer, J.M. and McMenamin, P.G. (2017). Where are we? The anatomy of the murine cortical meninges revisited for intravital imaging, immunology, and clearance of waste from the brain. Progress in neurobiology 156, 107-148.

7. Knopp, U., Christmann, F., Reusche, E. and Sepehrnia, A. (2005). A new collagen biomatrix of equine origin versus a cadaveric dura graft for the repair of dural defects--a comparative animal experimental study. Acta Neurochir (Wien) 147, 877-887.

8. Mirjana, P., Damir, S., Damir, J. and Martin, J. (2019). Biomechanical Comparison of the Temporalis Muscle Fascia, the Fascia Lata, and the Dura Mater. Journal of Neurological Surgery. Part B. Skull Base 80, 23-30.

9. Quaglini, V. and Villa, T. (2007). Mechanical properties of solvent-dehydrated bovine 
pericardium xenograft for dura mater repair. Journal of Applied Biomaterials and Biomechanics 5, 34-40.

10. Roth, T.L., Nayak, D., Atanasijevic, T., Koretsky, A.P., Latour, L.L. and McGavern, D.B. (2014). Transcranial amelioration of inflammation and cell death after brain injury. NATURE 505, 223228.

11. Maas, S.A., Erdemir, A., Halloran, J.P. and Weiss, J.A. (2016). A general framework for application of prestrain to computational models of biological materials. J. Mech. Behav. Biomed. Mater. 61, 499-510.

12. Ghajari, M., Hellyer, P.J. and Sharp, D.J. (2017). Computational modelling of traumatic brain injury predicts the location of chronic traumatic encephalopathy pathology. Brain 140, 333-343.

13. Hernandez, F., Giordano, C., Goubran, M., Parivash, S., Grant, G., Zeineh, M. and Camarillo, D. (2019). Lateral impacts correlate with falx cerebri displacement and corpus callosum trauma in sports-related concussions. Biomech. Model. Mechanobiology 18, 631-649.

14. Kleiven, S. (2007). Predictors for traumatic brain injuries evaluated through accident reconstructions. Stapp Car Crash J 51, 81-114.

15. Kleiven, S. (2013). Why Most Traumatic Brain Injuries are Not Caused by Linear Acceleration but Skull Fractures are. Frontiers in bioengineering and biotechnology 1, 15-15.

16. Gabler, L.F., Crandall, J.R. and Panzer, M.B. (2016). Assessment of Kinematic Brain Injury Metrics for Predicting Strain Responses in Diverse Automotive Impact Conditions. Ann Biomed Eng 44, 3705-3718.

17. MacManus, D.B., Pierrat, B., Murphy, J.G. and Gilchrist, M.D. (2017). Protection of cortex by overlying meninges tissue during dynamic indentation of the adolescent brain. Acta Biomaterialia 57, 384-394.

18. Prevost, T.P., Jin, G., de Moya, M.A., Alam, H.B., Suresh, S. and Socrate, S. (2011). Dynamic mechanical response of brain tissue in indentation in vivo, in situ and in vitro. Acta Biomaterialia 7, 4090-4101. 
19. Gu, L.X., Chafi, M.S., Ganpule, S. and Chandra, N. (2012). The influence of heterogeneous meninges on the brain mechanics under primary blast loading. Composites Part B-Engineering 43, 3160-3166.

20. Scott, G.G., Margulies, S.S. and Coats, B. (2016). Utilizing multiple scale models to improve predictions of extra-axial hemorrhage in the immature piglet. Biomech. Model. Mechanobiology $15,1101-1119$.

21. Scott, G.G. and Coats, B. (2015). Microstructural Characterization of the Pia-Arachnoid Complex Using Optical Coherence Tomography. leee Transactions on Medical Imaging 34, $1452-$ 1459.

22. Gu, L., Chafi, M.S., Ganpule, S. and Chandra, N. (2012). The influence of heterogeneous meninges on the brain mechanics under primary blast loading. Composites Part B: Engineering 43, 3160-3166.

23. Ho, J., Zhou, Z., Li, X. and Kleiven, S. (2017). The peculiar properties of the falx and tentorium in brain injury biomechanics. Journal of Biomechanics 60, 243-247.

24. Jacobson, S. and M. Marcus, E. (2011). Neuroanatomy for the Neuroscientist. Springer.

25. Protasoni, M., Sangiorgi, S., Cividini, A., Culuvaris, G.T., Tomei, G., Dell'Orbo, C., Raspanti, M., Balbi, S. and Reguzzoni, M. (2011). The collagenic architecture of human dura mater. J Neurosurg 114, 1723-1730.

26. Sacks, M.S., Hamann, M.C.J., Otaño-Lata, S.E. and Malinin, T.I. (1998). Local mechanical anisotropy in human cranial dura mater allografts. Journal of Biomechanical Engineering 120, 541. 27. Hamann, M.C., Sacks, M.S. and Malinin, T.I. (1998). Quantification of the collagen fibre architecture of human cranial dura mater. J Anat 192 ( Pt 1), 99-106.

28. Absinta, M., Ha, S.K., Nair, G., Sati, P., Luciano, N.J., Palisoc, M., Louveau, A., Zaghloul, K.A., Pittaluga, S., Kipnis, J. and Reich, D.S. (2017). Human and nonhuman primate meninges harbor lymphatic vessels that can be visualized noninvasively by MRI. eLife 6 .

29. Adeeb, N., Mortazavi, M.M., Tubbs, R.S. and Cohen-Gadol, A.A. (2012). The cranial dura 
mater: a review of its history, embryology, and anatomy. Child's Nervous System 28, 827-837. 30. Kriewall, T.J., Akkas, N., Bylski, D.I., Melvin, J.W. and Work, B.A. (1983). Mechanical behavior of fetal dura mater under large axisymmetric inflation. Journal of Biomechanical Engineering 105, 71-76.

31. Sellés de Lucas, V., Dutel, H., Evans, S.E., Gröning, F., Sharp, A.C., Watson, P.J. and Fagan, M.J. (2018). An assessment of the role of the falx cerebri and tentorium cerebelli in the cranium of the cat (Felis silvestris catus). J R Soc Interface 15.

32. Jeffery, N. (2002). Differential regional brain growth and rotation of the prenatal human tentorium cerebelli. J. ANAT. 200, 135-144.

33. Marín-Padilla, M. and Knopman, D.S. (2011). Developmental aspects of the intracerebral microvasculature and perivascular spaces: insights into brain response to late-life diseases. Journal of neuropathology and experimental neurology 70, 1060-1069.

34. Fabris, G., Suar, Z.M. and Kurt, M. (2019). Micromechanical heterogeneity of the rat piaarachnoid complex. Acta biomaterialia 100, 29-37.

35. Frantz, C., Stewart, K.M. and Weaver, V.M. (2010). The extracellular matrix at a glance. J Cell Sci 123, 4195-4200.

36. Ohtani, O. (1992). The maceration technique in scanning electron microscopy of collagen fiber frameworks: its application in the study of human livers. Archives of histology and cytology 55 Suppl, 225-232.

37. Walsh, D.R., Ross, A.M., Malijauskaite, S., Flanagan, B.D., Newport, D.T., McGourty, K.D. and Mulvihill, J.J.E. (2018). Regional mechanical and biochemical properties of the porcine cortical meninges. Acta Biomaterialia 80, 237-246.

38. McGarvey, K.A., Lee, J.M. and Boughner, D.R. (1984). Mechanical suitability of glycerolpreserved human dura mater for construction of prosthetic cardiac valves. Biomaterials 5, 109117.

39. van Noort, R., Black, M.M., Martin, T.R.P. and Meanley, S. (1981). A study of the uniaxial 
mechanical properties of human dura mater preserved in glycerol. Biomaterials 2, 41-45.

40. Maikos, J.T., Elias, R.A.I. and Shreiber, D.I. (2008). Mechanical properties of dura mater from the rat brain and spinal cord. Journal of Neurotrauma 25, 38-51.

41. Rai, R., Iwanaga, J., Shokouhi, G., Oskouian, R.J. and Tubbs, R.S. (2018). The Tentorium Cerebelli: A Comprehensive Review Including Its Anatomy, Embryology, and Surgical Techniques. Cureus 10, e3079-e3079.

42. Tanaka, Y. and Takeuchi, K. (1974). Dural Calification from the Neurosurgical Point of View. Neurol. Med.-Chir. 14pt1, 5-10.

43. Tatarli, N., Ceylan, D., Canaz, H., Tokmak, M., Bay, H.H., Seker, A., Keles, E., Kilic, T. and Cavdar, S. (2013). Falcine venous plexus within the falx cerebri: anatomical and scanning electron microscopic findings and clinical significance. Acta neurochirurgica 155, 2183-2189; discussion 2189-2189; discussion 2189.

44. Lee, S.-H., Shin, K.-J., Koh, K.-S. and Song, W.-C. (2020). Visualization of the tentorial innervation of human dura mater. J. ANAT. 231, 683-689.

45. Mortazavi, M.M., Quadri, S.A., Khan, M.A., Gustin, A., Suriya, S.S., Hassanzadeh, T., Fahimdanesh, K.M., Adl, F.H., Fard, S.A., Taqi, M.A., Armstrong, I., Martin, B.A. and Tubbs, R.S. (2018). Subarachnoid Trabeculae: A Comprehensive Review of Their Embryology, Histology, Morphology, and Surgical Significance. World Neurosurg 111, 279-290.

46. Benko, N., Luke, E., Alsanea, Y. and Coats, B. (2020). Spatial distribution of human arachnoid trabeculae. J. ANAT.

47. Zoghi-Moghadam, M. and Sadegh, A.M. (2009). Global/local head models to analyse cerebral blood vessel rupture leading to ASDH and SAH. Comput. Methods Biomech. Biomed. Eng. 12, $1-12$.

48. Reina, M.A., López, A., Dittmann, M. and De Andrés, J.A. (2015). Ultrastructure of Spinal Dura Mater. In: Atlas of Functional Anatomy for Regional Anesthesia and Pain Medicine: Human Structure, Ultrastructure and 3D Reconstruction Images. Reina, M.A., De Andrés, J.A., Hadzic, 
A., et al. (eds). Springer International Publishing, pps. 411-434.

49. Fung and C (1998). Biomechanics : Mechanical Properties of Living Tissues.

50. Rackl, M. (2015). Material testing and hyperelastic material model curve fitting for Ogden, Polynomial and Yeoh models.

51. Destrade, M., Saccomandi, G. and Sgura, I. (2017). Methodical fitting for mathematical models of rubber-like materials. Proceedings of the Royal Society A: Mathematical, Physical and Engineering Sciences 473, 20160811.

52. Ramião, N.G., Martins, P.S., Rynkevic, R., Fernandes, A.A., Barroso, M. and Santos, D.C. (2016). Biomechanical properties of breast tissue, a state-of-the-art review. Biomech. Model. Mechanobiology 15, 1307-1323.

53. McKee, C.T., Last, J.A., Russell, P. and Murphy, C.J. (2011). Indentation Versus Tensile Measurements of Young's Modulus for Soft Biological Tissues. Tissue Engineering. Part B, Reviews 17, 155-164.

54. Porter, M.M. and Niksiar, P. (2018). Multidimensional mechanics: Performance mapping of natural biological systems using permutated radar charts. PLOS ONE 13, e0204309-e0204309. 55. Guimarães, C.F., Gasperini, L., Marques, A.P. and Reis, R.L. (2020). The stiffness of living tissues and its implications for tissue engineering. Nature Reviews Materials 5, 351-370.

56. Biewener, A.A. (2008). Tendons and Ligaments: Structure, Mechanical Behavior and Biological Function. In: Collagen: Structure and Mechanics. Fratzl, P. (ed). Springer US: Boston, MA, pps. 269-284.

57. Wilson, A.M., McGuigan, M.P., Su, A. and van den Bogert, A.J. (2001). Horses damp the spring in their step. NATURE 414, 895-899.

58. Fratzl, P. (2008). Collagen: Structure and Mechanics. Springer US.

59. Yang, W., Sherman, V.R., Gludovatz, B., Schaible, E., Stewart, P., Ritchie, R.O. and Meyers, M.A. (2015). On the tear resistance of skin. Nature Communications 6, 6649-6649.

60. Jacquemoud, C., Bruyere-Garnier, K. and Coret, M. (2007). Methodology to determine failure 
characteristics of planar soft tissues using a dynamic tensile test. J Biomech 40, 468-475.

61. Moher, D., Liberati, A., Tetzlaff, J. and Altman, D.G. (2009). Preferred reporting items for systematic reviews and meta-analyses: the PRISMA statement. PLoS medicine 6, e1000097.

62. Sauleau, P., Lapouble, E., Val-Laillet, D. and Malbert, C.H. (2009). The pig model in brain imaging and neurosurgery. Animal 3, 1138-1151.

63. Hoffmann, A., Stoffel, M.H., Nitzsche, B., Lobsien, D., Seeger, J., Schneider, H. and Boltze,

J. (2014). The Ovine Cerebral Venous System: Comparative Anatomy, Visualization, and Implications for Translational Research. PLOS ONE 9, e92990-e92990.

64. Budday, S., Ovaert, T.C., Holzapfel, G.A., Steinmann, P. and Kuhl, E. (2019). Fifty Shades of Brain: A Review on the Mechanical Testing and Modeling of Brain Tissue. Archives of Computational Methods in Engineering.

65. Li, X.G., Sandler, H. and Kleiven, S. (2017). The importance of nonlinear tissue modelling in finite element simulations of infant head impacts. Biomech. Model. Mechanobiology 16, 823-840. 66. Galford, J.E. and McElhaney, J.H. (1970). A viscoelastic study of scalp, brain, and dura. Journal of Biomechanics 3, 211-221.

67. Wolfinbarger Jr, L., Zhang, Y., Adam, B.-L.T., Homsi, D., Gates, K. and Sutherland, V. (1994). Biomechanical aspects on rehydrated freeze-dried human allograft dura mater tissues. J Appl Biomater 5, 265-270.

68. Dunn, M.G. and Silver, F.H. (1983). Viscoelastic behavior of human connective tissues: Relative contribution of viscous and elastic components. Connect. Tissue Res. 12, 59-70.

69. De Kegel, D., Vastmans, J., Fehervary, H., Depreitere, B., Vander Sloten, J. and Famaey, N. (2018). Biomechanical characterization of human dura mater. J. Mech. Behav. Biomed. Mater. $79,122-134$.

70. Zwirner, J., Scholze, M., Waddell, J.N., Ondruschka, B. and Hammer, N. (2019). Mechanical Properties of Human Dura Mater in Tension-An Analysis at an Age Range of 2 to 94 Years. Scientific reports 9, 1-11. 
71. Noort, R.v., Martin, T.R.P., Black, M.M., Barker, A.T. and Montero, C.G. (1981). The mechanical properties of human dura mater and the effects of storage media. Clinical Physics and Physiological Measurement 2, 197-197.

72. Shulyakov, A.V., Cenkowski, S.S., Buist, R.J. and Del Bigio, M.R. (2011). Age-dependence of intracranial viscoelastic properties in living rats. J. Mech. Behav. Biomed. Mater. 4, 484-497.

73. Chauvet, D., Carpentier, A., Allain, J.M., Polivka, M., Crepin, J. and George, B. (2010). Histological and biomechanical study of dura mater applied to the technique of dura splitting decompression in Chiari type I malformation. Neurosurgical Review 33, 287-294.

74. Golman, A., Wickwire, A., Harrigan, T., Iwaskiw, A., Armiger, R. and Merkle, A. Hierarchical model validation of the falx cerebri and tentorium cerebelli.

75. Staquet, H., Francois, P.-M., Sandoz, B., Laporte, S., Decq, P. and Goutagny, S. (2020). Surface reconstruction from routine CT-scan shows large anatomical variations of falx cerebri and tentorium cerebelli. Acta Neurochirurgica.

76. Jin, X., Lee, J.B., Leung, L.Y., Zhang, L., Yang, K.H. and King, A.I. (2006). Biomechanical response of the bovine pia-arachnoid complex to tensile loading at varying strain-rates. Stapp Car Crash J 50, 637-649.

77. Jin, X., Ma, C., Zhang, L., Yang, K.H., King, A.I., Dong, G. and Zhang, J. (2007). Biomechanical response of the bovine pia-arachnoid complex to normal traction loading at varying strain rates. Stapp Car Crash J 51, 115-126.

78. Jin, X., Yang, K.H. and King, A.I. (2011). Mechanical properties of bovine pia \& arachnoid complex in shear. Journal of Biomechanics 44, 467-474.

79. Aimedieu, P. and Grebe, R. (2004). Tensile strength of cranial pia mater: Preliminary results. J. Neurosurg. 100, 111-114.

80. Fung (1998). Biomechanics : Mechanical Properties of Living Tissues.

81. Jin, X. (2009). Biomechanical response and constitutive modeling of bovine pia-arachnoid complex, Wayne State University; 2009. 
82. Hartmann, K., Stein, K.-P., Neyazi, B. and Sandalcioglu, I.E. (2019). First in vivo visualization of the human subarachnoid space and brain cortex via optical coherence tomography, pps. $1756286419843040-1756286419843040$.

83. MacManus, D.B., Pierrat, B., Murphy, J.G. and Gilchrist, M.D. (2017). Region and species dependent mechanical properties of adolescent and young adult brain tissue. Scientific Reports 7, 13729-13729.

84. Scott, G., Margulies, S. and Coats, B. (2016). Utilizing multiple scale models to improve predictions of extra-axial hemorrhage in the immature piglet. Biomechanics \& Modeling in Mechanobiology 15, 1101-1119.

85. Hoffman, A.H., Robichaud, D.R., 2nd, Duquette, J.J. and Grigg, P. (2005). Determining the effect of hydration upon the properties of ligaments using pseudo Gaussian stress stimuli. J Biomech 38, 1636-1642.

86. Meyer, J.P., McAvoy, K.E. and Jiang, J. (2013). Rehydration capacities and rates for various porcine tissues after dehydration. PLoS One 8, e72573.

87. Vilks, Y.K., Saulgozis, Y.Z. and Yanson, K.A. (1975). Effect of temperature on changes of tensile stress at constant elongation in certain human soft tissues. Polymer Mechanics 11, 780784.

88. Walsh, M.T., Cunnane, E.M., Mulvihill, J.J., Akyildiz, A.C., Gijsen, F.J.H. and Holzapfel, G.A. (2014). Uniaxial tensile testing approaches for characterisation of atherosclerotic plaques. Journal of Biomechanics 47, 793-804.

89. Henderson, J.H., Nacamuli, R.P., Zhao, B., Longaker, M.T. and Carter, D.R. (2005). Agedependent residual tensile strains are present in the dura mater of rats. J. R. Soc. Interface 2, 159-167.

90. Caro-Bretelle, A.S., Gountsop, P.N., lenny, P., Leger, R., Corn, S., Bazin, I. and Bretelle, F. (2015). Effect of sample preservation on stress softening and permanent set of porcine skin. Journal of Biomechanics 48, 3135-3141. 
91. Müller-Schweinitzer, E. (2009). Cryopreservation of vascular tissues. Organogenesis 5, 97104.

92. O'Leary, S.A., Doyle, B.J. and McGloughlin, T.M. (2013). Comparison of methods used to measure the thickness of soft tissues and their influence on the evaluation of tensile stress. $J$ Biomech 46, 1955-1960.

93. Zwirner, J., Ondruschka, B., Scholze, M., Schulze-Tanzil, G. and Hammer, N. (2019). Mechanical and morphological description of human acellular dura mater as a scaffold for surgical reconstruction. J. Mech. Behav. Biomed. Mater. 96, 38-44.

94. Zwirner, J., Scholze, M., Ondruschka, B. and Hammer, N. (2019). Tissue biomechanics of the human head are altered by Thiel embalming, restricting its use for biomechanical validation. Clinical Anatomy.

95. Holzapfel, G.A., Gasser, T.C. and Ogden, R.W. (2000). A New Constitutive Framework for Arterial Wall Mechanics and a Comparative Study of Material Models. Journal of elasticity and the physical science of solids $61,1-48$.

96. Tonge, T.K., Murienne, B.J., Coudrillier, B., Alexander, S., Rothkopf, W. and Nguyen, T.D. (2013). Minimal preconditioning effects observed for inflation tests of planar tissues. Journal of biomechanical engineering 135, 114502-114502.

97. Cooney, G.M., Moerman, K.M., Takaza, M., Winter, D.C. and Simms, C.K. (2015). Uniaxial and biaxial mechanical properties of porcine linea alba. J. Mech. Behav. Biomed. Mater. 41, 6882.

98. Mulvihill, J.J. and Walsh, M.T. (2013). On the mechanical behaviour of carotid artery plaques: the influence of curve-fitting experimental data on numerical model results. Biomech Model Mechanobiol 12, 975-985.

99. Zhou, B., Ravindran, S., Ferdous, J., Kidane, A., Sutton, M.A. and Shazly, T. (2016). Using Digital Image Correlation to Characterize Local Strains on Vascular Tissue Specimens. Journal of visualized experiments : JoVE 1, 53625-53625. 
100. Saboori, P. and Sadegh, A. (2015). Histology and Morphology of the Brain Subarachnoid Trabeculae. Anatomy Research International 2015, 1-9.

101. Scimeca, M., Bischetti, S., Lamsira, H.K., Bonfiglio, R. and Bonanno, E. (2018). Energy Dispersive X-ray (EDX) microanalysis: A powerful tool in biomedical research and diagnosis. European journal of histochemistry : EJH 62, 2841-2841.

102. Ho, J., Zhou, Z., Li, X.G. and Kleiven, S. (2017). The peculiar properties of the faix and tentorium in brain injury biomechanics. Journal of Biomechanics 60, 243-247.

103. Lind, N.M., Moustgaard, A., Jelsing, J., Vajta, G., Cumming, P. and Hansen, A.K. (2007).

The use of pigs in neuroscience: modeling brain disorders. Neurosci Biobehav Rev 31, 728-751. 104. Zhang, J., Yoganandan, N., Pintar, F.A. and Gennarelli, T.A. (2006). Brain strains in vehicle impact tests. Annu Proc Assoc Adv Automot Med 50, 1-12.

105. Hosseini-Farid, M., Amiri-Tehrani-Zadeh, M., Ramzanpour, M., Ziejewski, M. and Karami, G. (2020). The strain rates in the brain, brainstem, dura, and skull under dynamic loadings. Mathematical and Computational Applications 25, 21.

106. Rashid, B., Destrade, M. and Gilchrist, M.D. (2014). Mechanical characterization of brain tissue in tension at dynamic strain rates. J. Mech. Behav. Biomed. Mater. 33, 43-54.

107. Pervin, F. and Chen, W.W. (2009). Dynamic mechanical response of bovine gray matter and white matter brain tissues under compression. Journal of Biomechanics 42, 731-735.

108. Persson, C., Evans, S., Marsh, R., Summers, J.L. and Hall, R.M. (2010). Poisson's Ratio and Strain Rate Dependency of the Constitutive Behavior of Spinal Dura Mater. Ann Biomed Eng 38, 975-983.

109. Monea, A.G., Baeck, K., Verbeken, E., Verpoest, I., Sloten, J.V., Goffin, J. and Depreitere, B. (2014). The biomechanical behaviour of the bridging vein-superior sagittal sinus complex with implications for the mechanopathology of acute subdural haematoma. J. Mech. Behav. Biomed. Mater. 32, 155-165.

110. Yoganandan, N., Li, J.R., Zhang, J.Y., Pintar, F.A. and Gennarelli, T.A. (2008). Influence of 
angular acceleration-deceleration pulse shapes on regional brain strains. Journal of Biomechanics 41, 2253-2262.

111. Li, J., Zhang, J., Yoganandan, N., Pintar, F. and Gennarelli, T. (2007). Regional brain strains and role of falx in lateral impact-induced head rotational acceleration. Biomedical sciences instrumentation 43, 24-29.

112. Mao, H.J., Zhang, L.Y., Jiang, B.H., Genthikatti, V.V., Jin, X., Zhu, F., Makwana, R., Gill, A., Jandir, G., Singh, A. and Yang, K.H. (2013). Development of a Finite Element Human Head Model Partially Validated With Thirty Five Experimental Cases. Journal of Biomechanical EngineeringTransactions of the Asme 135.

113. Hardy, W.N., Mason, M.J., Foster, C.D., Shah, C.S., Kopacz, J.M., Yang, K.H., King, A.I., Bishop, J., Bey, M., Anderst, W. and Tashman, S. (2007). A study of the response of the human cadaver head to impact. Stapp Car Crash J 51, 17-80.

114. Hardy, W.N., Foster, C.D., Mason, M.J., Yang, K.H., King, A.I. and Tashman, S. (2001). Investigation of head injury mechanisms using neutral density technology and high-speed biplanar X-ray.

115. MacManus, D.B., Murphy, J.G. and Gilchrist, M.D. (2018). Mechanical characterisation of brain tissue up to $35 \%$ strain at 1, 10, and 100/s using a custom-built micro-indentation apparatus. J. Mech. Behav. Biomed. Mater. 87, 256-266.

116. Qian, L. and Zhao, H. (2018). Nanoindentation of Soft Biological Materials. Micromachines 9.

117. Cahalane, R.M. and Walsh, M.T. (2020). Nanoindentation of Calcified and Non-calcified Components of Atherosclerotic Tissues. Experimental Mechanics.

118. CDC (2003). Report to Congress on mild traumatic brain injury in the United States; steps to prevent a serious public health problem.

119. Voss, J.D., Connolly, J., Schwab, K.A. and Scher, A.I. (2015). Update on the Epidemiology of Concussion/Mild Traumatic Brain Injury. Current pain and headache reports 19, 32-32. 
120. Zhou, Z., Li, X. and Kleiven, S. (2020). Biomechanics of Acute Subdural Hematoma in the Elderly: A Fluid-Structure Interaction Study. Journal of Neurotrauma 36, 2099-2108.

121. Li, X. and Kleiven, S. (2018). Improved safety standards are needed to better protect younger children at playgrounds. Scientific Reports 8, 15061-15061.

122. Zhou, Z., Li, X. and Kleiven, S. (2019). Fluid-structure interaction simulation of the brain-skull interface for acute subdural haematoma prediction. Biomech. Model. Mechanobiology 18, 155173.

123. Holzapfel, G.A., Mulvihill, J.J., Cunnane, E.M. and Walsh, M.T. (2014). Computational approaches for analyzing the mechanics of atherosclerotic plaques: A review. Journal of Biomechanics 47, 859-869.

124. Miller, L.E., Urban, J.E. and Stitzel, J.D. (2016). Development and validation of an atlasbased finite element brain model. Biomech. Model. Mechanobiology 15, 1201-1214.

125. Gaul, R.T., Nolan, D.R. and Lally, C. (2017). Collagen fibre characterisation in arterial tissue under load using SALS. J. Mech. Behav. Biomed. Mater. 75, 359-368.

126. Chen, X., Nadiarynkh, O., Plotnikov, S. and Campagnola, P.J. (2012). Second harmonic generation microscopy for quantitative analysis of collagen fibrillar structure. Nature protocols 7 , 654-669.

127. Durgam, S., Singh, B., Cole, S.L., Brokken, M.T. and Stewart, M. (2020). Quantitative Assessment of Tendon Hierarchical Structure by Combined Second Harmonic Generation and Immunofluorescence Microscopy. Tissue Engineering Part C: Methods 26, 253-262.

128. Fung, Y.C. (1967). Elasticity of soft tissues in simple elongation. American Journal of Physiology-Legacy Content 213, 1532-1544. 


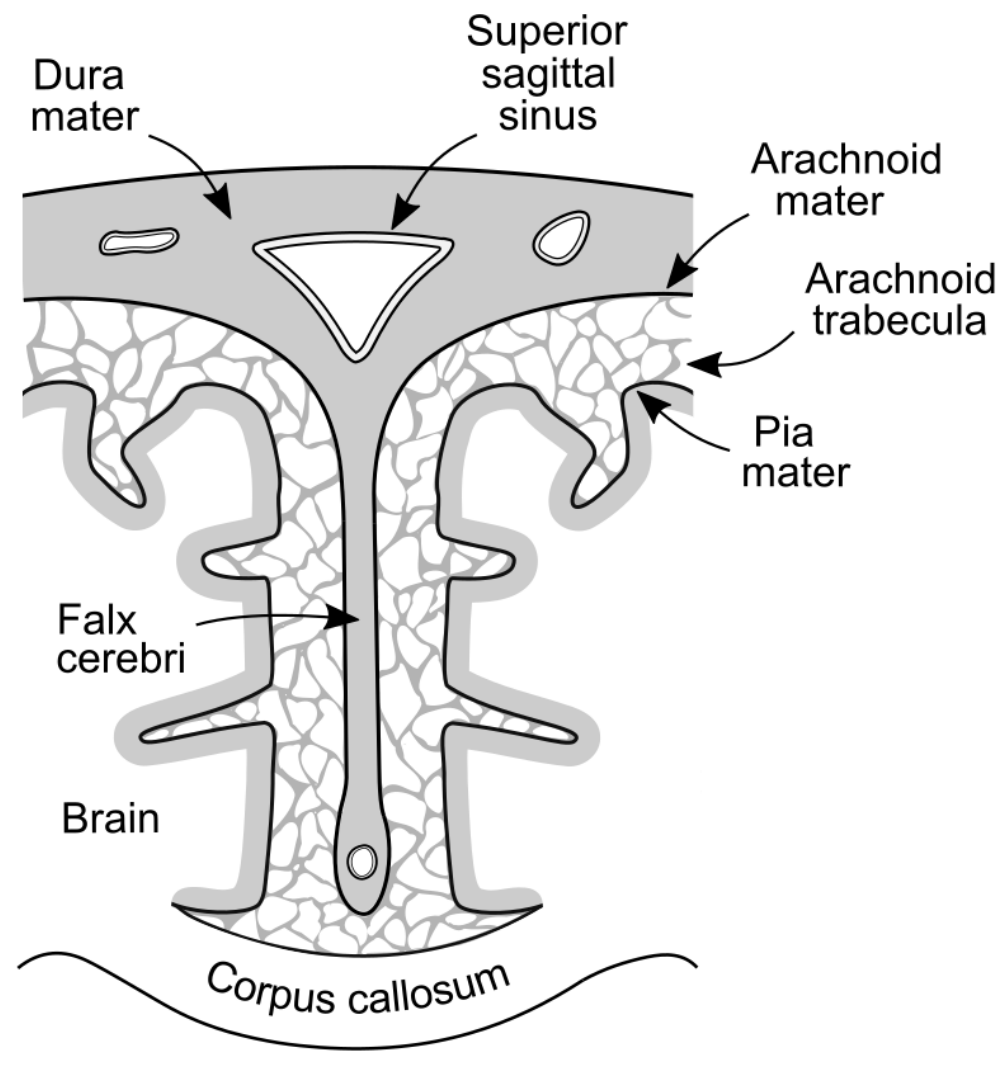

Fig. 1: (A) Illustration of meningeal anatomy including the dura mater, superior sagittal sinus, piaarachnoid complex, falx cerebri and corpus callosum. (B) Illustration demonstrating the region of interest depicted in (A). Note that the various regions are not drawn to scale. 


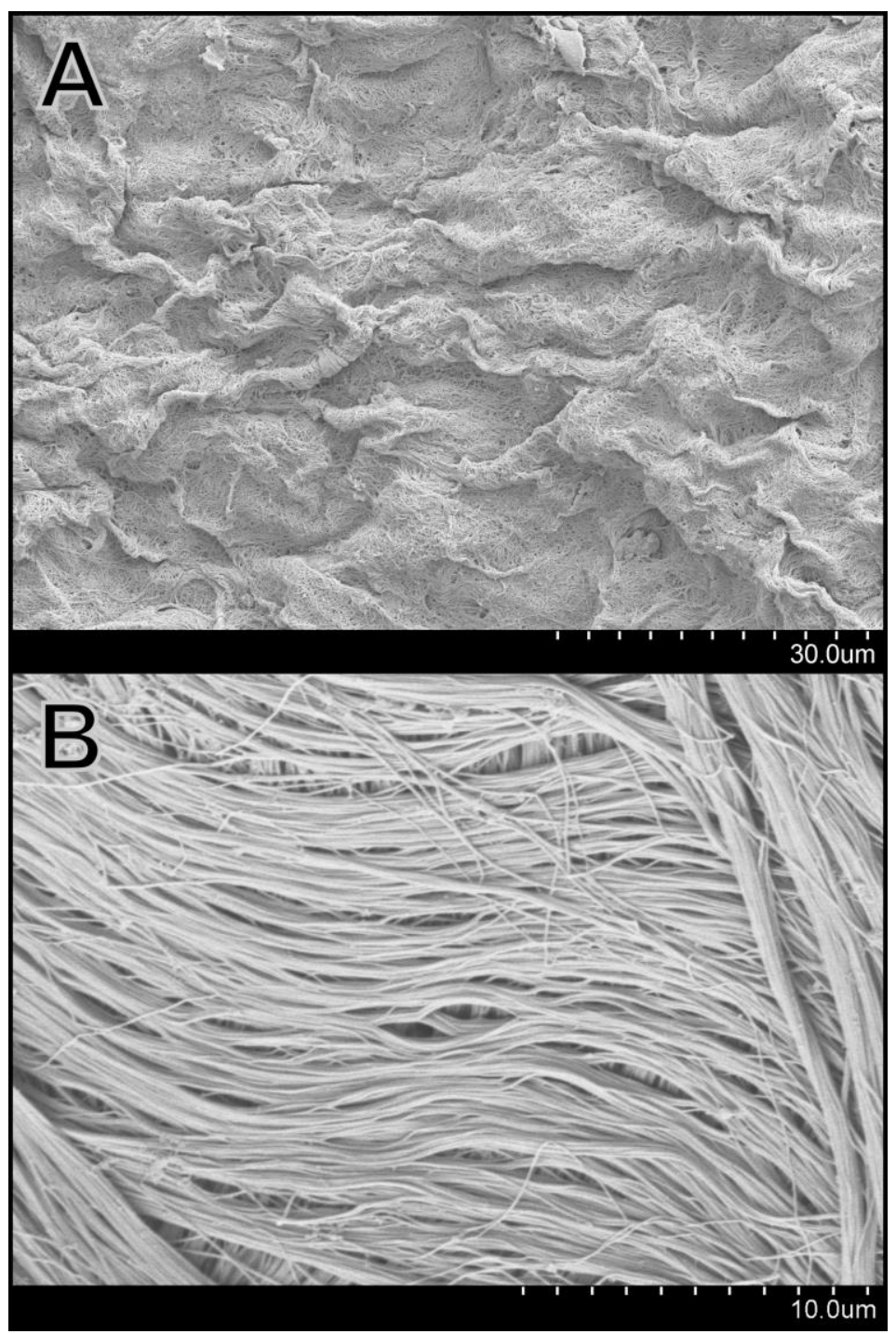

Fig. 2: Scanning electron microscopy image of macerated dura mater tissue demonstrating the dense collagenic architecture of the tissue. (A) The arachnoid surface layer (B) The bone surface layer. 


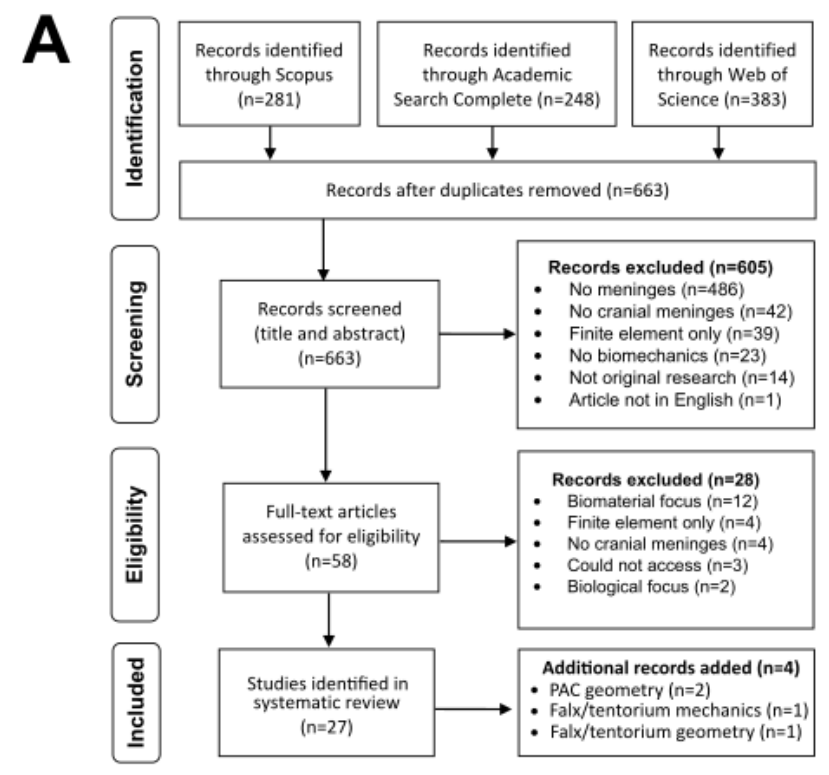

C Modality of testing; dura mater



B Identified studies by region



D Modality of testing; PAC



Fig. 3: (A) Workflow diagram summarising the identification of articles utilising three separate search platforms; Scopus, Academic Search Complete and Web of Science. (B) Summary of papers identified in the systematic review sorted by meningeal regions. No peer-reviewed studies were identified for falx cerebri and tentorium cerebelli mechanics. (C) Breakdown of testing modality employed in the studies which focussed on dura mater mechanics. (D) Breakdown of testing modality employed in the studies which focussed on pia-arachnoid complex (PAC) mechanics. AFM denotes atomic force microscopy. All studies which focussed on characterisation of whole meninges employed indentation testing. 
A Elastic modulus values for PAC, falx/tentorium and dura



B Species tested; dura mater



C Species tested; PAC

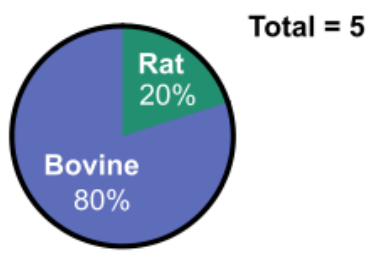

Fig. 4: (A) Summary of quasi-static elastic moduli results from the identified studies. Note that the modulus value assigned to the dura, falx and tentorium in the majority of FE models, based on the work of Galford et al (1970) on human dura mater tissue, is highlighted for comparison purposes. Data are presented as mean \pm s.d. Note that the reported elastic moduli were calculated from the linear region of the stress strain curve (i.e. after the initial 'toe-region') and were reported by the original study authors. (B) Breakdown of species tested in mechanical studies focusing on the cranial dura mater. (C) Breakdown of species tested in mechanical studies focussing on the cranial PAC. 
A Thickness values for tentorium, falx and dura



B Measurement technique utilised
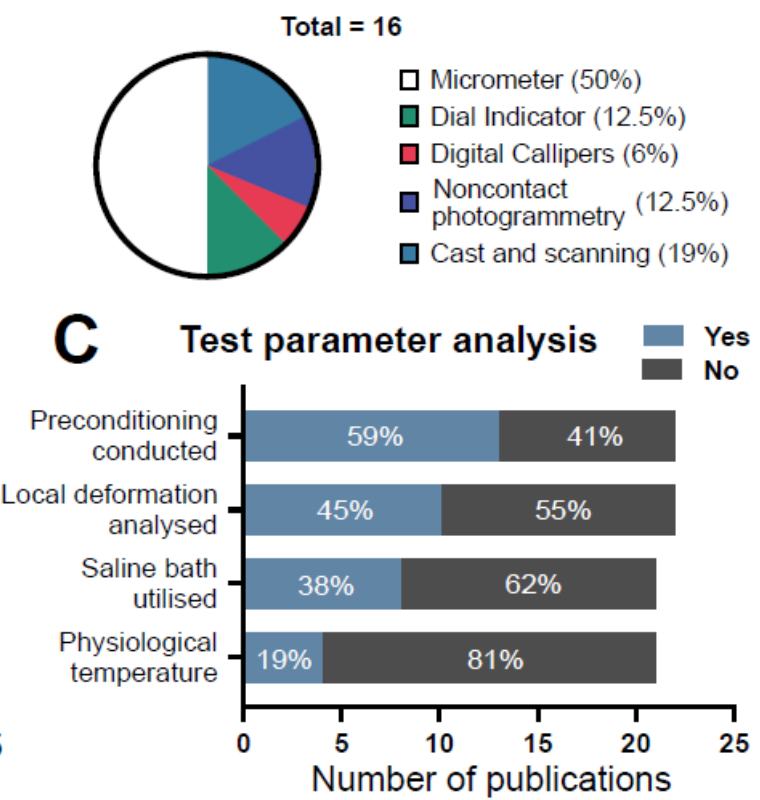

Fig. 5: (A) Thickness data from studies on human and porcine dura mater along with human falx and tentorium tissues. Values are presented as mean \pm s.d. Note that both Sacks $\left(1998^{*}\right)$ results pertain to two samples within the same study. PAC thickness values are not graphed due to the significantly smaller scale of the PAC. (B) Breakdown of measurement techniques utilised to analyse thickness in the identified studies. (C) Analysis of various test parameters in the identified studies such as whether sample preconditioning was conducted or not, if local sample deformation was analysed during sample characterisation, if test samples were submerged in a saline bath during testing to ensure tissue hydration and whether or not samples were tested at physiological temperature. 


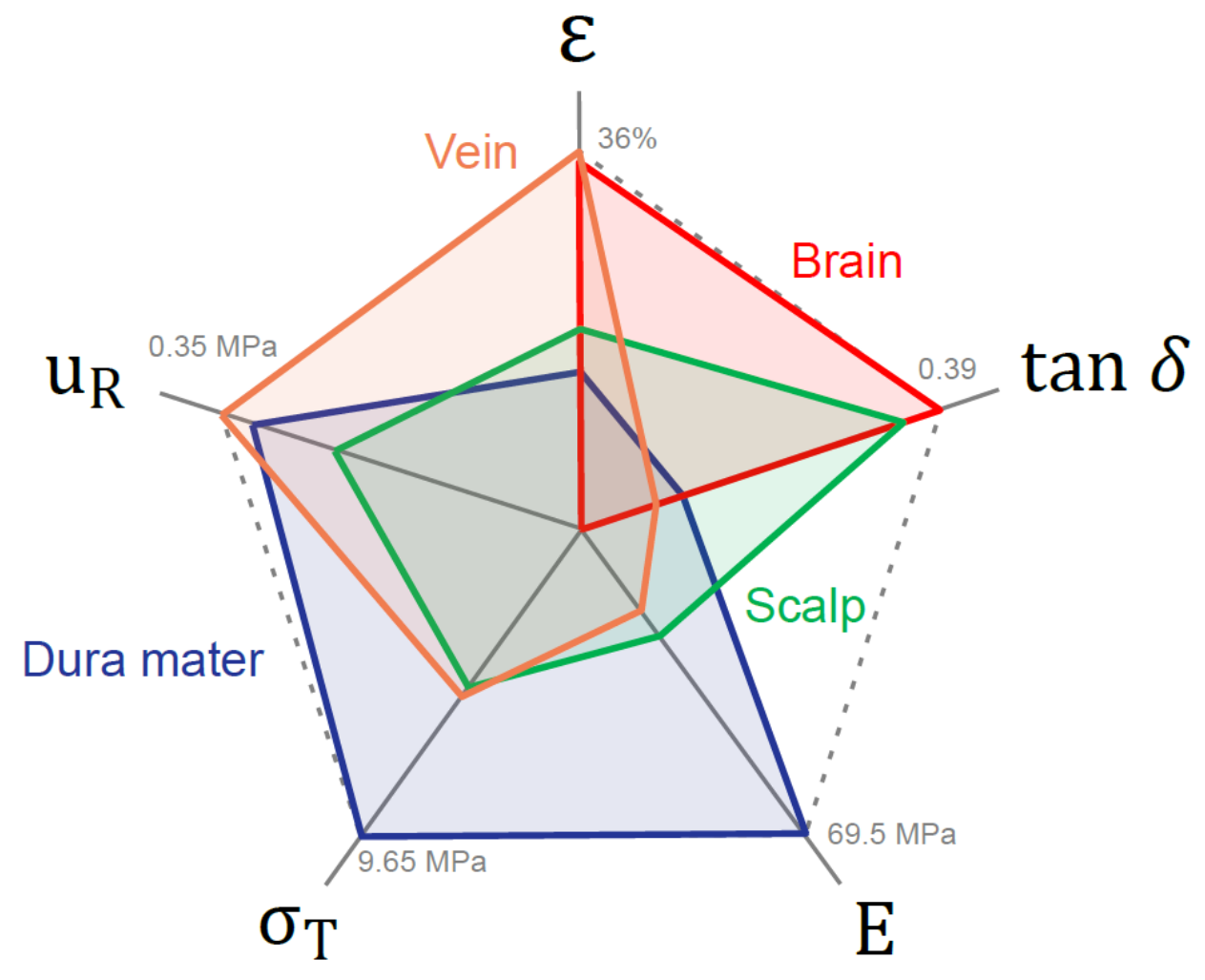

Fig. 6: Normalised, permuted radar plot comparing dura mater mechanical characteristics to other soft biological tissues including brain, venous tissue and scalp. Legend: strain to failure, $\epsilon$ (\%); damping loss factor, $\tan \delta$ (no units), elastic modulus, $E(\mathrm{MPa})$, tensile strength, $\sigma_{T}$, resilience, $\mathrm{u}_{\mathrm{R}}(\mathrm{MPa})$. Note that the maximum value for each averaged parameter is presented on the axes. Data presented in radar plot is as described in supplementary information table S7. 